\title{
Development of the Very Low Angle Detector (VLAD) for detection of epithermal neutrons at low momentum transfers
}

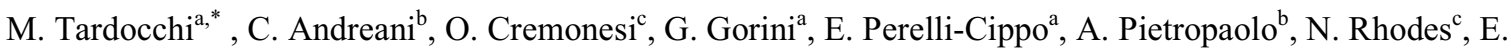 \\ Schooneveld ${ }^{\mathrm{c}}$, R. Senesi ${ }^{\mathrm{b}}$ \\ ${ }^{a}$ INFM, UdR Milano-Bicocca and Dipartimento di Fisica “G.Occhialini”, Università degli Studi di Milano- \\ Bicocca, Milano, Italy \\ ${ }^{\mathrm{b}}$ Dipartimento di Fisica, Università degli Studi di Roma Tor Vergata and INFM, UdR Roma Tor Vergata, Roma, \\ Italy \\ ${ }^{c}$ INFN, Sezione di Milano and Dipartimento di Fisica "G.Occhialini”, Università degli Studi di Milano-Bicocca, \\ Milano, Italy \\ ${ }^{\mathrm{d}}$ ISIS Facility, Rutherford Appleton Laboratory, Chilton, Didcot, UK
}

New perspectives for epithermal neutron spectroscopy are opened up by the recent development of new instrumentation for inverse geometry time of flight spectrometers at pulsed neutron sources. One example is the Very Low Angle Detector (VLAD) bank which will be installed as an upgrade of the VESUVIO neutron spectrometer, at the ISIS pulsed neutron source. VLAD is developed for detecting epithermal neutrons in the 1$100 \mathrm{eV}$ energy range at very low scattering angles $\left(1^{\circ}-5^{\circ}\right)$. VLAD will extend the kinematical region covered by today's neutron scattering experiments to the region of low wave vector transfers $\left(<10 \AA^{-1}\right)$ and high energy transfers $(>1 \mathrm{eV})$. Accessing such kinematical region will allow new experimental studies in condensed matter systems. The neutron detection is based on Resonance Detectors (RD), which consist of the combination of a resonance foil used as neutron-to-gamma converter and a photon detector. The results obtained with a prototype VLAD detector confirm the potential of this kind of experiments at scattering angles as low as $2^{\circ}-5^{\circ}$. GEANT4 simulations are used to address issues, such as detector cross talk, which arise with the construction of compact RD arrays.

Kewords: Neutron spectroscopy; epithermal neutrons; resonance detector; YAP

\section{INTRODUCTION}

The recent development of new instrumentation for inverse geometry time of flight spectrometers at pulsed neutron sources is motivated by the interest to extend the kinematical region which can be covered by today's neutron scattering experiments. In particular, by accessing the kinematical region of low wave vector transfer $\left(\mathrm{q}<10 \AA^{-1}\right)$ and, at the same time, high energy transfer $(\hbar \omega>1 \mathrm{eV})$, new experimental studies in condensed matter systems are possible [1]. In order to access this kinematical region the detection of epithermal neutrons in the 1$100 \mathrm{eV}$ energy range at scattering angles below $5^{\circ}[2]$ is required.

Scattering experiments with epithermal neutrons are today routinely performed on the inverse geometry neutron spectrometer VESUVIO [3], installed at the ISIS pulsed neutron source. The neutron detection technique employed, the so called Resonance Filter Technique, is based on ${ }^{6} \mathrm{Li}$ glass scintillators for detection of the scattered neutrons that are transmitted through an analyzer foil [4]. The scattering time of flight spectrum at the final neutron energy specified by the capture resonance is obtained through the difference of the measurements with and without the analyzer foil. This detection technique becomes inefficient at neutron energies $\left(\mathrm{E}_{\mathrm{n}}\right)$ above, say, $20 \mathrm{eV}$ due to the $1 / \sqrt{ } E_{n}$ dependence of the neutron capture cross-section of ${ }^{6} \mathrm{Li}$. The Resonance Detector (RD) has been developed for scattering experiments at these neutron energies.

The RD [5] consists of an analyser foil $\left({ }^{A} \mathrm{X}\right)$ attached to a photon detector. Neutron detection is

${ }^{*}$ Corresponding author. Tel.: +39-02-6448.2326; fax: +39-02-6448.2367; e-mail: Marco.Tardocchi@mib.infn.it. 
performed in a two-step process: i) resonant neutron capture by the analyzer foil which strongly absorbs the scattered neutrons over a narrow energy interval; ii) detection of the prompt radiative capture $\gamma$-rays (as well as of the associated X-rays) following the radiative decay of the compound nucleus $\left({ }^{\mathrm{A}+1} \mathrm{X}\right)$ to the ground state. The function of the photon detector is to record the arrival time of the absorbed neutron required for the time of flight technique.

One of the first applications of the RD is the realization of the Very Low Angle Detector (VLAD) array on the VESUVIO spectrometer. The usefulness of the RD for neutron scattering experiment at very low angles was tested in measurements with four VLAD prototype detectors at scattering angles as low as $2^{\circ}-5^{\circ}$ The results obtained are presented in this work. The paper addresses issues studied with GEANT4 simulations, such as detector cross talk and multiple neutron scattering, which arise with the construction of compact RD arrays. The outcome of the simulations will be used as input to the optimization of the final VLAD design.

\section{THE OPTIMIZED RD}

VESUVIO [3] is an inverse geometry neutron spectrometer installed at the ISIS spallation neutron source. On this instrument (Fig. 1) a white neutron beam impinges on the sample, placed inside an aluminum vacuum chamber. Scattered neutrons are transmitted through an analyser foil $\left({ }^{238} \mathrm{U}\right.$ or $\left.{ }^{197} \mathrm{Au}\right)$ which absorbs neutron of selected energies. The transmitted neutrons are detected with ${ }^{6} \mathrm{Li}$ glass scintillators located in both forward and backscattering positions. An additional developed vacuum tank is installed in order to minimize the amount of material intercepted by the neutrons scattered at very low angles. The tank is $2 \mathrm{~m}$ long and terminates with a $1 \mathrm{~mm}$ thick aluminum flange behind which four RD prototype units of the VLAD array are located. Each RD unit consists of a ${ }^{238} \mathrm{U}$ analyzer foil and a photon detector.

The RD has been developed over the past few years by testing different combinations of analyzer foils and photon detectors [6]. The ideal analyzer foil should have widely separated resonances in the 1-100 $\mathrm{eV}$ energy range, with large cross sections and narrow energy widths. High resonance cross sections allow for good neutron-to- $\gamma$ conversion efficiency with thin analyser foils (thickness of the order of few tens of microns) which have a low self absorption probability of the prompt radiative capture $\gamma$ and $X$ rays. Narrow resonance widths are needed as they are often the main contribution to the energy resolution of the spectrometer. The best material is ${ }^{238} \mathrm{U}$, which has several narrow resonances in the $1-100 \mathrm{eV}$ energy range [7]. The radiative capture $\gamma$-ray emission from ${ }^{238} \mathrm{U}$ consists of many lines ranging from $11 \mathrm{keV}$ up to $4.060 \mathrm{MeV}$ [8]. The ideal photon detector should have good efficiency to the prompt radiative capture $\gamma$-rays while being insensitive to other background

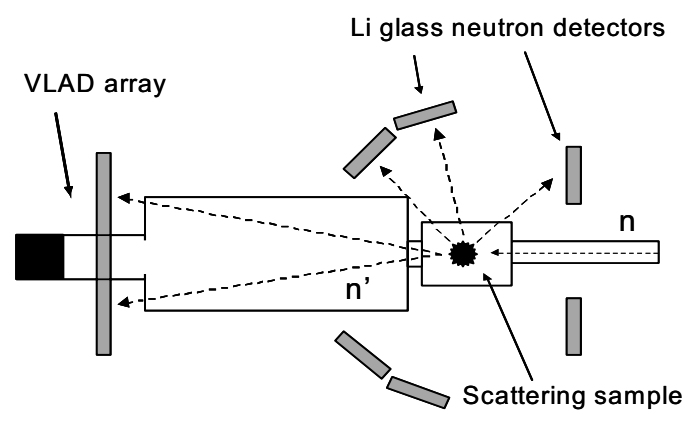

Fig. 1 Schematics of the VESUVIO spectrometer showing the incoming neutron beam from the right (n), the scattering sample, the vacuum tank, the scattered neutrons (n') and the detector positions. The VLAD array is located in the forward position.

radiation, mostly represented by environmental $\gamma$-rays and neutrons. The performance of different photon detectors have been tested [9-11] Good results were obtained with CZT detectors which could detect epithermal neutrons of energies up to $70 \mathrm{eV}$ with a signal to background ratio (S/B) exceeding conventional ${ }^{6} \mathrm{Li}$ glass scintillators [12]. Recently, the $\mathrm{RD}$ performance was further improved by the use of $\mathrm{YAlO}_{3}$ (YAP) scintillators. Compared to the CZT detector YAP scintillators offer the advantage of a lower cost per detector unit area and the possibility of larger detector sizes. YAP is an inorganic scintillator with a good light yield $(\approx 18000$ photons $/ \mathrm{MeV})$ and a short decay time $(\forall=27 \mathrm{~ns})$. The chosen crystals had a thickness of $6 \mathrm{~mm}$ which represented a good compromise between the need for good detection efficiency to the radiative capture $\gamma$ emission and low neutron scattering in the crystal. The response of the YAP scintillator to radiative capture emission from a ${ }^{238} \mathrm{U}$ analyzer foil, investigated in a set of biparametric - $\gamma$ pulse height and neutron time of flight - measurements, has shown that the signal is predominantly made up of Compton events induced by the higher energy (above $700 \mathrm{keV}$ ) radiative capture $\gamma$ rays which are not fully stopped in the 
detector [8]. The quality of the time of flight measurement depends on the relative intensity of the signal peaks to the background, i.e. the signal to background ratio. Figure 2 shows that by raising the lower level discrimination on the $\gamma$ energy from 40

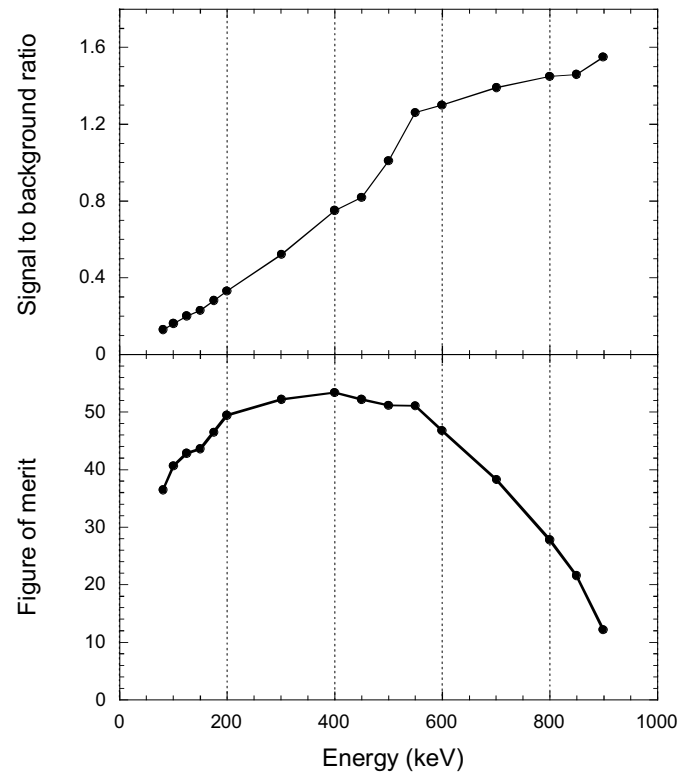

Fig. 2 Signal to background ratio (S/B, top) and Figure of merit (bottom) versus the lower level discrimination on the $\gamma$ energy for the ${ }^{238} \mathrm{U}$ resonance located at $20.9 \mathrm{eV}$.

show a similar trend. On the base of a figure of merit of the achievable statistical uncertainty in the signal peaks, defined in Ref. [8], it was shown that the best results are reached with the threshold set in the energy range $400-600 \mathrm{keV}$ (see Figure 2). The improvement in $\mathrm{S} / \mathrm{B}$ that is achieved by raising the lower level discrimination is due to the discrimination of the background source represented by radiative capture $\gamma$-rays in boron (at $478 \mathrm{keV}$ ). The latter is commonly used as neutron shielding material [8].

A lower level discrimination of $400-600 \mathrm{keV}$ would not appear ideal from the $\gamma$ detection point of view since the YAP detector thickness is optimized for detecting photons below $200 \mathrm{keV}$. However, a thicker crystal providing a higher detection efficiency would also increase the level of neutron backscattering in the YAP crystal itself. Neutron backscattering can cause a distortion of shape of the signal peak which is where the physics information of scattering experiments lies. Simulations are at present in progress in order to quantify the neutron backscattering level and to find the optimum
$\mathrm{keV}$ to $600 \mathrm{keV}$ a significant improvement in $\mathrm{S} / \mathrm{B}$ can be achieved. These data were obtained using a lead scattering sample and refer to the ${ }^{238} \mathrm{U}$ resonance located at $20.9 \mathrm{eV}$ [8]. The other ${ }^{238} \mathrm{U}$ resonances

thickness of the YAP crystal, representing a trade off between $\gamma$ detection efficiency and neutron backscattering effects.

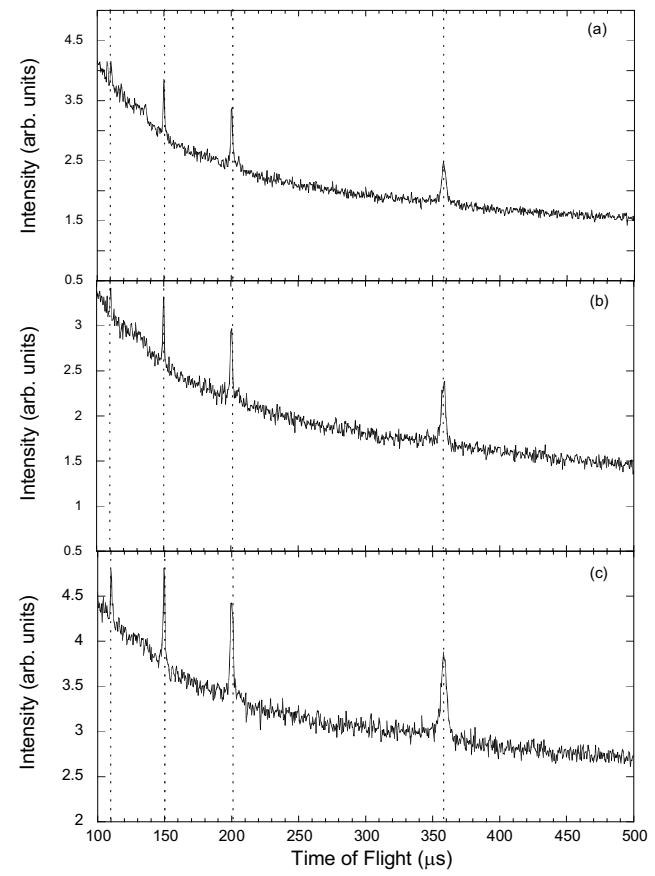

Fig. 3 Time of flight spectra registered from an ice sample in an Al cell in the time region 50-500 $\mu$ s. The spectra were recorded at scattering angles of $2^{\circ}$ (a), $3.5^{\circ}$ (b) and $5^{\circ}$ (c). The dashed lines indicate the $\mathrm{H}$ recoil peaks corresponding to the first four ${ }^{238} \mathrm{U}$ resonances $\left(\mathrm{E}_{\mathrm{n}}=6.67 \mathrm{eV}, 20.9 \mathrm{eV}, 36.7 \mathrm{eV}\right.$ and $\left.66.0 \mathrm{eV}\right)$.

\section{VLAD PROTOTYPE}

The VLAD bank of the VESUVIO spectrometer is planned to cover the $1^{\circ}-5^{\circ}$ angular range at a distance of about $2 \mathrm{~m}$ from the scattering sample. A detector bank prototype was built to demonstrate the feasibility of RD at very low scattering angles. The prototype consisted of four RD units, each made from two $6 \mathrm{~mm}$ thick YAP crystals of trapezoidal shape attached to $25 \mu \mathrm{m}{ }^{238} \mathrm{U}$ analyser foils of the same sizes. The crystals were enclosed in an Aluminium casing acting as a lightguide towards a single photomultiplier tube. The four RD were positioned at angles between $2^{\circ}$ and $5^{\circ}$ and measured neutrons scattered from an ice sample at $270 \mathrm{~K}$. The corresponding time of flight spectra are shown in Fig. 
3. The four peaks clearly visible in all spectra are due to hydrogen recoil peaks at the first four neutron resonances of ${ }^{238} \mathrm{U}$. An analysis of these data has shown that it is possible to derive physical quantities

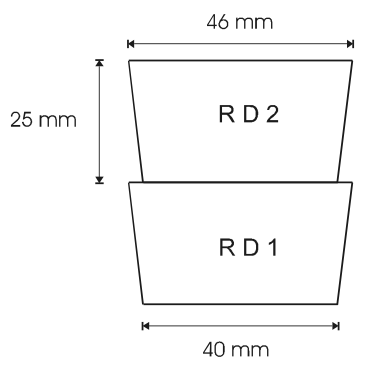

Fig. 4 Detector sizes and positions used in the GEANT4 simulations.

different instrument [13].

The favorable results obtained with the prototype VLAD detectors indicate the feasibility of a VLAD array based on the RD. The VLAD bank design is at present being finalised for scattering measurements at angles in the range $1^{\circ}-5^{\circ}$. One issue to be considered in the optimisation of the VLAD geometry is the cross talk among different detector channels. A cross talk event can be due, for instance, to a radiative capture $\gamma$ which makes a Compton interaction in the first RD (called RD1) and a second interaction in a neighbouring RD (called RD2). This effect has been simulated with GEANT4 [14] for the detector geometry shown in Figure 4 where the RD shape is the same as the one of the VLAD prototype crystals. The spectrum of the energy deposited in RD1 by a $4.06 \mathrm{MeV} \gamma$ isotropically emitted from a ${ }^{238} \mathrm{U}$ analyser foil attached to RD1 is shown in Figure 5. Note that the broadening contribution due to the intrinsic energy resolution of the YAP detector has not been considered. The spectrum is mostly flat due to Compton interactions extending up to the Compton edge located at $3.82 \mathrm{MeV}$. On the top of that clearly visible are the full energy peak and the single and double escape peaks. The neighboring detector RD2 sees a certain amounts of (cross talk) events, equal to $6 \%$ of the total events occurring in RD1. The simulated cross talk spectrum (Figure 5) has a continuous distribution but with events pushed towards lower energies, beside the double escape and annihilation peaks. The total amount of recorded cross talk events can be reduced by applying a lower level discrimination. In the example of Figure 5 the such as the dynamical structure factor and the density of states of hydrogen in the sample, in agreement with earlier measurements performed on a

cross talk level is reduced to $<2.5 \%$ by applying a $600 \mathrm{keV}$ threshold. This is an acceptable cross talk level, especially considering that the cross talk decreases slightly with decreasing energies of the prompt radiative capture $\gamma$ 's.

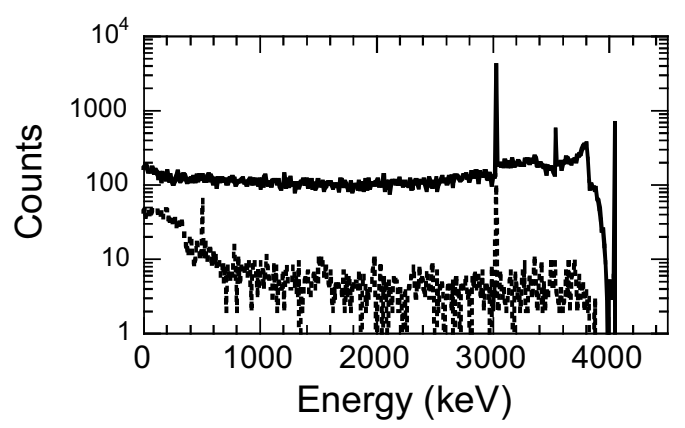

Fig. 5 Simulated spectrum of the energy deposited (continuous line) by $4.060 \mathrm{MeV} \gamma$ interactions in RD1. In dashed line it is shown the (cross-talk) spectrum of the energy deposited by the same photon in RD2.

\section{CONCLUSIONS}

New perspectives for epithermal neutron spectroscopy are opened up by the recent development of the Resonant Detector (RD) at the VESUVIO neutron spectrometer on ISIS. The reference RD consists of the combination of a ${ }^{238} \mathrm{U}$ analyser foil and a YAP photon detector.

The first application of the RD is the Very Low Angle Detector (VLAD) bank, which will be installed on VESUVIO. VLAD will extend the kinematical region for neutron scattering to low wave vector transfer $\left(\mathrm{q}<10 \AA^{-1}\right)$ and, at the same time, high energy transfer $(\hbar \omega>1 \mathrm{eV})$, allowing new experimental investigations in condensed matter system. The first results obtained with a prototype VLAD detector bank have indicated the feasibility of the RD for scattering measurements at angles as low as $2^{\circ}-5^{\circ}$.

The VLAD design is at the moment being finalised, with the help of GEANT4 simulations addressing issues such as detector cross talk and multiple neutron scattering. The simulations have shown that the cross talk level can be kept below $2.5 \%$ by using a suitable lower level discrimination 


\section{AKNOWLEDGMENTS}

Work performed with financial support by the European Community-Access to Research Infrastructure action of the Improving Human Potential Programme We acknowledge Consiglio Nazionale delle Ricerche (CNR)-Italy for financial support for the experiments performed at the ISIS pulsed neutron source.

\section{REFERENCES}

[1] R. Newport et al., Nucl. Instr. and Meth. 224(1984) 120.

[2] S. Imberti et al., "Resolution of a low angle epithermal neutrons spectrometer", manuscript in preparation.

[3] R. Senesi, C. Andreani, Z. Bowden, D. Colognesi, E. Degiorgi, A.L. Fielding, J. Mayers, M. Nardone, J. Norris, M. Praitano, N.J. Rhodes, W.G. Stirling, J. Tomkinson, C. Uden, Physica B 276-278, (2000) 200.

[4] A. L. Fielding and J. Majers, Nucl. Instr. and Meth. A48(2002) 680.

[5] D.R. Allen, E.W.J. Mitchell, R.N. Sinclair, J. Phys. E Sci. Instrum. 13, (1980) 639; J.M. Carpenter, N. Watanabe, S. Ikeda, Y. Masuda, S. Sato, Physica B120, (1983) 126.

[6] G. Gorini et al, "The resonant detector and its application to epithermal neutron spectroscopy", to appear on Nucl. Instr. and Meth. A.

[7] A. Pietropaolo, C. Andreani, A. D’Angelo, G. Gorini, S. Imberti, N. Rhodes, E. Schooneveld, R. Senesi, M. Tardocchi, Appl. Phys. A 74, (2002) S189.

[8] M. Tardocchi et al., "YAP scintillators for resonant detection of epithermal neutrons at pulsed neutron sources", submitted to Review of Scientific Instruments.

[9] C. Andreani, A. Pietropaolo, R. Senesi, G. Gorini, M. Tardocchi, A. Bracco, N. Rhodes, E. Schooneveld, Nucl. Instr. and Meth. A 481 (2002) 509.

[10] M. Tardocchi, C. Arnaboldi, G. Gorini, S. Imberti, G. Pessina, E. Previtali, C. Andreani, A. Pietropaolo, R. Senesi, “Assessment of a silicon detector for pulsed neutron scattering experiments", to appear on Physica B.

[11] M. Tardocchi, A. Pietropaolo, C. Andreani, A. Bracco, A. D'Angelo, G. Gorini, S. Imberti, R. Senesi, N. Rhodes, E. S. Schooneveld, Nucl. Instr. and Meth. A, 526/3 (2004) 279.

[12] C. Andreani, A. D'Angelo, G. Gorini, S. Imberti, A.Pietropaolo, N. Rhodes, E.Schooneveld, R. Senesi, M.Tardocchi, Appl. Phys. A 78, 903 (2004).

[13] E. Perelli-Cippo et al., "First results of the Very Low Angle Detector bank prototype on VESUVIO", manuscript in preparation.

[14] S. Agostinelli, J. Allison, K. Amako, J. Apostolakis, H. Araujo, P. Arce, M. Asai, D. Axen, S. Banerjee, G. Barrand et al., Nucl. Instr. and Meth. A, 506(2003) 250. 